\section{Atypical gastric presentation of strongyloidiasis in an immunecompetent patient}


Fig. 1 a Upper endoscopic view of the gastric antrum showing multiple subepithelial hemorrhages. b Endoscopic findings in the duodenum: edema, erythematous spots, small ulcers, loss of vascular pattern, thickened folds, and mucosal erosions. The pathology report showed eggs, larvae and adult forms of Strongyloides stercoralis in the antrum (c) and the duodenum (d).



Fig. 2 Endoscopic view of the gastric antrum (a) and the duodenum (b) after 6 weeks of treatment with ivermectin, showing resolution of lesions.
A 52-year-old female presented with a 4-year history of epigastric pain, nausea, vomiting, and watery diarrhea without mucus or blood. She received medication including lanzoprazol, and antidiarrhea and antispasmodic treatments without improvement. Her medical history was unremarkable, with no hospital admissions or surgical procedures. Vital signs were normal. Physical examination revealed tenderness in the colonic area with normal bowel sounds. No signs of peritoneal irritation were detected. Abdominal ultrasound was normal. Laboratory tests reported eosinophilia (9\%). HIV test was negative. Normal lymphocyte subpopulations were present. No parasites were found in repeated fecal smears.

Upper endoscopy demonstrated multiple subepithelial hemorrhages in the gastric antrum ( Fig.1 a), and edema, erythematous spots, small ulcers, loss of vascular pattern, thickened folds, and mucosal erosions in the duodenum ( Fig. 1b). Biopsy samples were taken from the antrum and duodenum. The presence of eggs, larvae, and adult forms of Strongyloides stercoralis was evident (๑ Fig. 1 c, d).

Treatment with albendazole, $400 \mathrm{mg}$, was indicated twice a day for 3 days within 3 weeks [1]. A reduction in subepithelial hemorrhages, edema, and ulcers was observed 6 weeks later but parasites were still detected. Nitazoxanide was then initiated, but it was not well tolerated due to vomiting. Therefore, two doses of ivermectin, $200 \mu \mathrm{g} / \mathrm{kg} /$ day, were indicated [2], resulting in a remarkable improvement of symptoms, including resolution of gastric and duodenum lesions; histology was negative for parasites 6 weeks after treatment ( $\bullet$ Fig. 2 a, b).

The patient has remained asymptomatic after 1 year of follow-up. Gastric involvement in strongyloidiasis has been rarely reported but reduced gastric acid secretion might favor infection and invasion of the stomach [3], via consequent sputum swallowing or retrograde migration from the proximal small intestine [4]. Although this patient was immunecompetent, with a low risk for S. stercoralis infection, the history of long-term medication with proton pump inhibitors, could be considered a predisposing condition allowing the parasite to access the stomach. 
Endoscopy_UCTN_Code_CCL_1AB_2AD_3AC

Endoscopy_UCTN_Code_CCL_1AB_2AD_3AF

H. Montes ${ }^{1}$, A. Arenas ${ }^{2}$, P. Petrosino ${ }^{2}$, M. Milano ${ }^{2}$, S. Salmen ${ }^{3}$, L. Berrueta ${ }^{3}$

1 Gastroenterology Unit, CAMIULA, Los Andes University, Mérida, Venezuela

2 Department of Pathology, Los Andes University, Mérida, Venezuela

3 Clinical Immunology Institute, Los Andes University, Mérida, Venezuela

\section{References}

1 Keiser PB, Nutman TB. Strongyloides stercoralis in the immunocompromised population. Clin Microbiolog Rev 2004; 17: 208 - 217

2 Albonico M, Crompton DW, Savioli L. Control strategies for human intestinal nematode infections. Adv Parasitol 1999; 42: 277-341

3 Gianella RA, Broitman SA, Zamchek N. Influence of gastric acidity on bacterial and parasitic enteric infections. A perspective. Ann Intern Med 1973; 78: 271 - 276

$4 \mathrm{Kim}$ J, Joo HS, Kim DH et al. A case of gastric strongylodiasis in a Korean patient. Korean J Parasitol 2003; 41: 63-67
Bibliography

DOI 10.1055/s-2007-995497

Endoscopy 2008; 40: E230 - E231

(c) Georg Thieme Verlag KG Stuttgart · New York . ISSN 0013-726X

Corresponding author

\section{H. Montes, $M$}

Gastroenterology Unit

CAMIULA

University of Los Andes

Av Urdaneta

Mérida

Venezuela

Fax: + 58-274-2403187

montesh@yahoo.com 